\title{
Determination of the oxidation state and coordination of a vanadium doped chalcogenide glass
}

\author{
Mark A. Hughes \\ Department of Quantum Engineering, Nagoya University, Furo-cho, Chikusa-ku, Nagoya \\ 464-8603, Japan
}

Richard J. Curry

Advanced Technology Institute, University of Surrey, Guildford, Surrey GU2 7XH, UK

\section{Daniel W. Hewak}

Optoelectronics Research Centre, University of Southampton, Southampton SO17 1BJ, United Kingdom

Vanadium doped chalcogenide glass has potential as an active gain medium, particularly at telecommunications wavelengths. This dopant has three spin allowed absorption transitions at 1100, 737 and $578 \mathrm{~nm}$, and a spin forbidden absorption transition at 1000 $\mathrm{nm}$. X-ray photo electron spectroscopy indicated the presence of vanadium in a range of oxidation states from $\mathrm{V}^{+}$to $\mathrm{V}^{5+}$. Excitation of each absorption band resulted in the same characteristic emission spectrum and lifetime, indicating that only one oxidation state is optically active. Arguments based on Tanabe-Sugano analysis indicated that the configuration of the optically active vanadium ion was octahedral $\mathrm{V}^{2+}$. The calculated crystal field parameters $(\mathrm{Dq} / \mathrm{B}, \mathrm{B}$ and $\mathrm{C} / \mathrm{B})$ were $1.85,485.1$ and 4.55 , respectively.

\section{Introduction}

Determination of the oxidative state of an active ion dopant is important for optical device applications as it determines the energy levels within the material available for use. Knowledge of the oxidation state is therefore needed when modelling the radiative and non-radiative transitions that occur in an optical material. The oxidation states of transition metal ions are particularly difficult to identify by spectroscopy as their bonding $\mathrm{d}$ electrons also determine their electronic energy levels and they are therefore strongly dependent on both the strength and the arrangement (coordination) of the neighbouring atoms electric field. In contrast, rare-earth metals, in which the electronic energy levels are determined by the $4 f$ electrons which do not take part in bonding and are shielded by the $5 s 5 p$ electrons, the oxidation state can be identified relatively easily by spectroscopy. Amorphous glass hosts, with their tendency to result in mixed oxidation states due to significant variation in the local environment, lead to a complex superposition of electronic states when doped, which exacerbates the problem of spectroscopic analysis of transition metals.

Chalcogenide glasses often exhibit low phonon energy and this allows the observation of optical transitions in dopants that are not observed in traditional glasses such as silica. Gallium lanthanum sulphide (GLS) has a transmission window of $\sim 0.5-10$ 
$\mu \mathrm{m}[1]$, a high refractive index of $\sim 2.4$, and a low maximum phonon energy of $\sim 425 \mathrm{~cm}^{-1}$ which results in low non-radiative decay rates [2]. These properties allowed the observation of low-energy transitions which are not seen in other hosts, for example the first observation of the $4.9 \mu \mathrm{m}$ fluorescence from the ${ }^{5} \mathrm{I}_{4} \rightarrow{ }^{5} \mathrm{I}_{5}$ transition of $\mathrm{Ho}^{3+}$ [3]. Also, rarely observed $\mathrm{Ti}^{3+}$ emission [4] and long-wavelength emission from $\mathrm{Bi}$ [5] have both also been reported from GLS host glasses.

The emission from transition metal doped glasses often overlaps the technologically useful low-loss window of silica optical fibers $\sim 1300-1700 \mathrm{~nm}$, also known as the 'telecommunications window'. Therefore various transition metal doped glasses have been proposed as gain media for devices that could enable the utilization of bandwidth in the telecommunications window not currently covered by the erbium-doped fiber amplifier (EDFA). To date these studies have mainly focused on $\mathrm{Cr}$ - and Ni-doped glasses [6-8] with very little work exploring V-doped glasses as active gain media. In previous work we suggested that V-doped GLS (V:GLS) could be a potential gain media covering the telecommunications window, because it displays photoluminescence peaking at $1500 \mathrm{~nm}$ with a full width at half maximum (FWHM) of $500 \mathrm{~nm}$ [9]. We also suggested that the vanadium configuration was tetrahedral $\mathrm{V}^{3+}$. In this work we revise this to octahedral $\mathrm{V}^{2+}$, based mainly on the consideration of further possible configurations and a more detailed analysis using the Tanabe-Sugano model.

\section{Experimental}

Samples of V:GLS were prepared by mixing $65 \%$ gallium sulphide $(\mathrm{GaxS}), 30 \%$ lanthanum sulphide $\left(\mathrm{La}_{2} \mathrm{~S}_{3}\right), 5 \%$ lanthanum oxide $\left(\mathrm{La}_{2} \mathrm{O}_{3}\right)$, and $0.01-1 \%$ vanadium sulphide $\left(\mathrm{V}_{2} \mathrm{~S}_{3}\right)(\mathrm{mol}$. \%) in a dry-nitrogen purged glovebox. Melt components were batched into vitreous carbon crucibles and weighed using a balance with a resolution of $0.001 \mathrm{~g}$. Batches were then transferred to a furnace using a custom built sealed atmosphere transfer pod. Gallium and lanthanum sulphides were synthesised in-house from gallium metal ( $9 \mathrm{~N}$ purity) and lanthanum fluoride ( $5 \mathrm{~N}$ purity) precursors in a flowing $\mathrm{H}_{2} \mathrm{~S}$ gas system. The precise stoichiometry of the gallium sulphide was not known as some sulphur was lost during melting, however we believe that $\mathrm{x}=1.4$ based on measurements of mass loss. Prior to sulphide synthesis, lanthanum fluoride was purified and dehydrated in a dry-argon purged furnace at $1250{ }^{\circ} \mathrm{C}$ for 36 hours to reduce $\mathrm{OH}^{-}$and transition metal impurities. The lanthanum oxide and vanadium sulphide were purchased commercially and used without further purification. The glass was melted at $1150{ }^{\circ} \mathrm{C}$ for around 24 hours, in a silica tube furnace, with an initial ramp rate of $20{ }^{\circ} \mathrm{C}$ $\min ^{-1}$ and under a constant argon atmosphere (flow of $200 \mathrm{ml} \mathrm{min}^{-1}$ ). This method was chosen in favour of the sealed ampoule method because volatile impurities such as $\mathrm{OH}^{-}$ are carried downstream away from the melt and because of safety concerns of the ampoules exploding. The melt was rapidly quenched (at around $500{ }^{\circ} \mathrm{C} \mathrm{min}{ }^{-1}$ ) to form a glass by pushing the crucible holder into a water-cooled silica jacket. The quenching process is designed to prevent crystallisation of the glass by rapidly increasing the viscosity of the glass through rapid temperature drop, hence arresting the nucleation and growth of crystals. The glass was then annealed at $500{ }^{\circ} \mathrm{C}$ for 12 hours resulting in 
homogeneous and crack free samples. Samples were cut and polished into $5 \mathrm{~mm}$ thick slabs for characterization.

Absorption spectra were obtained using a Varian Cary 500 spectrophotometer over a range of 175-3300 nm with a resolution of $\pm 0.1 \mathrm{~nm}$. Photoluminescence (PL) spectra were obtained by dispersing fluorescence, excited using 514, 808, and $1064 \mathrm{~nm}$ laser lines, in a Bentham TMc300 monochromator and detecting the output using liquid nitrogen cooled $\mathrm{InSb}$ or InGaAs detector coupled with standard phase sensitive detection. All spectra were corrected for the system response. To obtain photoluminescence excitation (PLE) spectra, a $1400 \mathrm{~nm}$ long pass filter was placed in front of an InGaAs detector to give an effective detection range of 1400-1700 nm. A $250 \mathrm{~W}$ quartz halogen white light source passing through a monochromator provided the excitation source with a $5 \mathrm{~nm}$ bandwidth. The PLE spectra were corrected for the varying intensity of exciting light due to varying grating response and spectral output of the white light source by characterizing the output of each grating with wavelength calibrated Newport 818-SL and 818-IG detectors and a Newport 1830-c optical power meter. Fluorescence lifetime measurements were obtained using a $1064 \mathrm{~nm} \mathrm{Nd:YAG} \mathrm{laser} \mathrm{modulated} \mathrm{using} \mathrm{a} \mathrm{Gooch}$ and Housego $80 \mathrm{MHz}$ acousto-optic modulator, with the fluorescence detected using a New Focus 2053 InGaAS detector.

For X-ray photoelectron spectroscopy (XPS) measurements a sample of $1 \mathrm{~mol}$. \% $\mathrm{V}$ :GLS glass was fractured to reveal a flat face with dimensions of around $5 \mathrm{~mm} \times 5 \mathrm{~mm}$, this was then placed into the vacuum chamber of an XPS system. The vacuum chamber was evacuated to around $10^{-9}$ mbar and the sample was exposed to X-ray radiation from an $\mathrm{Mg} \mathrm{K} \alpha$ anode source centred at $1253.6 \mathrm{eV}$. The full width at half maximum (FWHM) of the anode source limited the resolution of the photoelectron spectra to $1 \mathrm{eV}$.

\section{Results}

\subsection{Absorption}

Fig. 1 shows the absorption spectrum of $0.1 \% \mathrm{~V}$ :GLS and undoped GLS, the second differential $\left(\mathrm{d}^{2} \alpha / \mathrm{d} \lambda^{2}\right)$ of these spectra is also shown. The undoped absorption spectrum is typical of GLS, showing a strong electronic absorption edge at $\sim 500 \mathrm{~nm}$. Within the absorption spectrum of the $0.1 \% \mathrm{~V}$ :GLS sample, a broad absorption centred at $\sim 1100 \mathrm{~nm}$ and a shoulder at $\sim 750 \mathrm{~nm}$ can be identified. The red-shift of the absorption edge in the doped glass indicates that a third vanadium absorption band lies close to the un-doped GLS absorption edge $\sim 500 \mathrm{~nm}$.

The mathematical differentiation of spectroscopic data is often used as a resolution enhancement technique, to facilitate the detection and location of poorly resolved spectral components including peaks which appear only as shoulders as well as the isolation of small peaks from an interfering large background absorption [10]. In second differential spectra, absorption bands correspond to negative peaks below $\mathrm{d}^{2} \alpha / \mathrm{d} \lambda^{2}$ $=0$. In the $\mathrm{d}^{2} \alpha / \mathrm{d} \lambda^{2}$ spectrum of $0.1 \% \mathrm{~V}$ :GLS in Fig. 1, the previously identified absorption band at $1100 \mathrm{~nm}$ clearly visible, as is the shoulder at $\sim 750 \mathrm{~nm}$, which can now be specified more precisely to $737 \mathrm{~nm}$. However, an absorption band not apparent in the absorption spectrum is clearly visible at $1000 \mathrm{~nm}$ in the differentiated spectrum. The second differential of the undoped GLS absorption spectrum does not show any features, 
indicating that the all of the features in the differentiated V:GLS spectrum are due to the presence of vanadium, and not the host glass or artefacts of the measurement system. Since the $1000 \mathrm{~nm}$ absorption band was only apparent in the differentiated spectrum we can infer that this absorption band is much narrower than the other absorption bands because higher order derivatives discriminate strongly in favour of narrower bands [11]. By examining the Tanabe-Sugano diagrams in Fig. 4 it can be seen that energy levels with the same spin as the ground state have a strong dependence on crystal field strength, whereas energy levels with different spin to the ground state generally have almost zero dependence on crystal field strength. In most host materials, especially glasses, there is a broad range of crystal field strengths, therefore, spin-allowed transitions result in relatively strong, broad absorption bands and broad emission bands with short lifetimes $(\sim \mathrm{ns}$ to $\mu \mathrm{s})$. Whereas spin-forbidden transitions result in relatively weak, narrow absorption bands and narrow emission bands with long lifetimes ( $\sim \mathrm{ms}$ to $\mathrm{s})$. We can therefore infer that the $1000 \mathrm{~nm}$ absorption band results from a spin forbidden transition.

\subsection{Photoluminescence and photoluminescence excitation spectra}

Fig 2 shows the PLE spectra of $0.1 \%$ vanadium doped GLS. The PLE spectrum shows three broad absorption bands, characteristic of spin allowed transitions. An excellent fit of the PLE spectrum is obtained with three Gaussians (labelled a, b and c) as shown. The peaks of Gaussians a, b and c were at 578, 766 and $1154 \mathrm{~nm}$, respectively. The 766 and $1154 \mathrm{~nm}$ absorption bands identified from the PLE spectrum in Fig 2 clearly relate to the 737 and $1100 \mathrm{~nm}$ absorption bands identified by differential absorption in Fig. 1.

The PL spectra of $0.1 \% \mathrm{~V}$ :GLS in Fig 2 shows that excitation at 514, 808 and $1064 \mathrm{~nm}$ results in broad emission peaking at 1480, 1510 and $1470 \mathrm{~nm}$, respectively. We propose that the shift to longer wavelengths of the PL peak upon $808 \mathrm{~nm}$ excitation is due to preferential excitation of ions in lower crystal field strength sites, noting that this excitation energy is lower energy than the $737 \mathrm{~nm}$ absorption band. This could also explain why the 766 and $1154 \mathrm{~nm}$ absorption bands identified by PLE measurements in Fig. 2 are at longer wavelengths than the 737 and $1100 \mathrm{~nm}$ absorption bands identified by absorption measurements in Fig. 1. This is because less absorption of PL occurs when the PL peaks at longer wavelengths, so the PLE peak will be at longer wavelengths than the absorption peak. The excitation wavelengths used to obtain the PL spectra in Fig 2 roughly equate to exciting each of the three main absorption bands identified in the PLE spectrum. The PL from excitation at these wavelengths show similar characteristic spectra, with a full width at half maximum FWHM of $\sim 500 \mathrm{~nm}$, they also all have lifetimes of $\sim 30 \mu \mathrm{s}$ [9]. This indicates that the three absorption bands belong to the same oxidation state, rather than two or more oxidation states which is commonly observed in transition metal doped glasses [12-14] and crystals [15]. The broadness of the PL spectra and its short lifetime indicates that the vanadium ion is in a low crystal field site, where the lowest energy transition is a spin-allowed transition. Conversely, in a strong crystal field site the lowest spin-forbidden level (which is almost independent of crystal field strength) is the lowest energy level. In this case, characteristic narrow $R$-line emission should be observed, as in $\mathrm{V}^{3+}$ doped phosphate glass [16] and $\mathrm{V}^{3+}$ doped corundum [17]. 


\subsection{X-ray photoelectron spectroscopy}

An XPS spectrum of 1\% vanadium doped GLS is shown in Fig. 3 with the inset showing the vanadium peak in more detail. Photoelectron spectra of non-conducting samples are known to have a shift in energy due to charging of the sample which can exert an attractive force on escaping photoelectrons and hence cause an unknown energy shift in the spectra [18]. This is usually corrected for from the position of the C1s peak of nonintrinsic carbon present in nearly all samples [19]. However, the region of the C1s signal showed a complex structure, making it difficult to assign the peak of the adventitious carbon, hence the spectrum was corrected using the O1s peaks. The elastic tail, or Shirley background, due to electron scattering off ion sites, was removed as standard. Following these procedures the spectrum was deconvolved using a series of Gaussian-Lorentzian peaks into a best-fit of the measured spectrum.

The spectra in Fig. 3 show a very broad vanadium peak suggesting the presence of mixed oxidation states $\mathrm{V}^{5+} / \mathrm{V}^{4+} / \mathrm{V}^{3+} / \mathrm{V}^{2+} / \mathrm{V}^{0+}$, with $\mathrm{V}^{5+}$ being the dominant species. However, the resolution and signal strength of the measurement is not high enough to unambiguously identify each oxidation state and give an accurate compositional ratio. A mixture of vanadium oxidation states has been observed in other glasses, as follows. This is not unexpected given the nature of amorphous materials. Optical analysis indicated the presence of $\mathrm{V}^{5+}, \mathrm{V}^{4+}$ and $\mathrm{V}^{3+}$ in vanadium doped flame-hydrolysed fused silica [20] and vanadium doped $\mathrm{Na}_{2} \mathrm{O} .2 \mathrm{SiO}_{2}$ glass [13]. The labelling of the deconvolved peaks for each of the different vanadium oxidation states is consistent with XPS spectra previously taken of $\mathrm{V}_{2} \mathrm{O}_{5}, \mathrm{VO}_{2}, \mathrm{~V}_{2} \mathrm{O}_{3}$ and $\mathrm{V}$ metal, which we attributed to $2 \mathrm{p}^{3 / 2}$ core electrons [21]. The peak at $520 \mathrm{eV}$ is consistent with the $2 \mathrm{p}^{1 / 2}$ peak of vanadium [21]. XPS has been used to determine the oxidation state of chromium doped sodium silicate glass [12], which similarly showed a mixture of oxidation states present in the form of $\mathrm{Cr}^{2+}, \mathrm{Cr}^{3+}$ and $\mathrm{Cr}^{6+}$. In glass melts containing multiple vanadium oxidation states, the oxidation states usually interact as redox pairs such as $\mathrm{V}^{3+} / \mathrm{V}^{4+}$ and $\mathrm{V}^{4+} / \mathrm{V}^{5+}$ [22]. Based on the redox reactions of vanadium oxides in silicate glasses [23], we propose the following redox reactions for the transformation of the $\mathrm{V}_{2} \mathrm{~S}_{3}\left(\mathrm{~V}^{3+}\right)$ starting material into $\mathrm{V}^{2+}, \mathrm{V}^{4+}$ and $\mathrm{V}^{5+}$ in Eqs. (1), (2) and (3), respectively.

$$
\begin{aligned}
& V^{3+}+\frac{3}{2} S^{2-} \Leftrightarrow V S+\frac{1}{4} S_{2} \\
& V^{3+}+\frac{1}{2} S^{2-}+\frac{1}{4} S_{2} \Leftrightarrow(V S)^{2+} \\
& V^{4+}+\frac{1}{2} S^{2-}+\frac{1}{4} S_{2} \Leftrightarrow(V S)^{3+}
\end{aligned}
$$

The four O1s peaks in Fig. 3 give an indication of the structure of GLS glass. The lowest binding energy peak at $530 \mathrm{eV}$ is tentatively assigned to a non-bridging oxygen i.e. Ga-O ${ }^{2-}$ of the oxide negative cavities of GLS described in ref [24]. This is because the effective charge on the oxygen atom in the oxide negative cavity environment will be higher than that of a bridging oxygen, and an increase in electron density on the relevant 
atom screens the core electrons and hence decreases the measured binding energy [25]. The other three peaks at 534, 537 and $539 \mathrm{eV}$ are attributed to bridging bonds of La-OLa, Ga-O-Ga and S-O-S, respectively.

\section{Discussion of each possible vanadium oxidation state}

Vanadium 5+ has a $\mathrm{d}^{0}$ electronic configuration and will not have any $\mathrm{d}-\mathrm{d}$ optical transitions. However, $d^{0}$ ions can contribute to optical transitions if there is a chargetransfer process. Charge-transfer transitions are usually high energy transitions which are predicted by molecular orbit theory but not by crystal field theory. These high-energy transitions promote electrons that mainly belong to states of ligand ions to states that mainly belong to the transition metal ion [26]. Charge-transfer transitions of $\mathrm{V}^{5+}$ in various glass and crystal hosts all occur in or around the UV region [13, 27, 28]. Since the optical transitions observed for vanadium doped GLS occur at lower energies they are not attributed to a charge-transfer transition. Therefore $\mathrm{V}^{5+}$ is not believed to contribute to any observed optical transition of V:GLS.

Vanadium $4+$ has a $\mathrm{d}^{1}$ electronic configuration which means there is only one excited $E_{g}$ state [29] and therefore it has only one spin-allowed absorption transition $\left({ }^{2} \mathrm{~T}_{2} \rightarrow{ }^{2} \mathrm{E}_{2}\right)$. Vanadium $4+$ doped $\mathrm{CaYAlO}_{4}$ displays a broad absorption band at $500 \mathrm{~nm}$ [30]. Two excitation peaks for $\mathrm{V}^{4+}$ have been observed due to the Jan-Teller effect at 427 and $490 \mathrm{~nm}$ in $\mathrm{Al}_{2} \mathrm{O}_{3}$, at 419 and $486 \mathrm{~nm}$ in $\mathrm{YAlO}_{3}$ and at 432 and $500 \mathrm{~nm}$ in yttrium aluminium garnet (YAG) [29]. Based on these comparisons it is thought to be unlikely that $\mathrm{V}^{4+}$ can account for the three broad absorption bands observed in V:GLS.

Vanadium $3+$ has a $\mathrm{d}^{2}$ electronic configuration and, from inspection of the Tanabe-Sugano diagram for a $\mathrm{d}^{2}$ ion [31-33], is expected to have three spin-allowed ground-state absorption transitions in both tetrahedral and octahedral coordination. Tetrahedral $\mathrm{V}^{3+}$ in YAG has three absorption bands, centred at 600,800 and $1320 \mathrm{~nm}$, a weak and narrow absorption at $1140 \mathrm{~nm}$ is attributed to a spin-forbidden transition [15, 34, 35]. Octahedral $\mathrm{V}^{3+}$ in $\mathrm{Na}_{2} \mathrm{O} \cdot 2 \mathrm{SiO}_{2}$ glass displays two absorption peaks, at 690 and $450 \mathrm{~nm}$, [13]. Therefore, $3+$ is a possible oxidation state of vanadium in GLS.

Vanadium $2+$ has a d $\mathrm{d}^{3}$ electronic configuration and from inspection of the TanabeSugano diagram for a $\mathrm{d}^{3}$ ion [31-33] is also expected to have three spin-allowed groundstate absorption transitions when in octahedral coordination. In the case of a tetrahedrally coordinated $\mathrm{d}^{3}$ ion there are three spin-allowed transitions in weak crystal fields $(\mathrm{Dq} / \mathrm{B}<2.2)$, and four spin-allowed transitions in strong crystal files $(\mathrm{Dq} / \mathrm{B}>2.2)$. In octahedral $\mathrm{V}^{2+}$ doped $\mathrm{MgF}_{2}$ there are three spin-allowed transition absorption bands centred at 884, 550 and $366 \mathrm{~nm}$, a spin-forbidden transition was also observed at $787 \mathrm{~nm}$ [36]. The low temperature $(\mathrm{T}=10 \mathrm{~K})$ absorption spectra of octahedral $\mathrm{V}^{2+}$ doped $\mathrm{NaCl}$ has spin-allowed transition absorption bands, centred at 1222, 759 and $478 \mathrm{~nm}$ [37]. Octahedral $\mathrm{V}^{2+}$ doped $\mathrm{CsCaF}_{3}$ has spin-allowed transition absorption bands centred at 1067, 662 and $424 \mathrm{~nm}$, a spin-forbidden transition was also observed at $794 \mathrm{~nm}$ and [38]. Based on these observations, 2+ is also a possible oxidation state of vanadium in GLS.

Vanadium $1+$ has a $\mathrm{d}^{4}$ electronic configuration and inspection of the TanabeSugano diagram for a $\mathrm{d}^{4}$ ion [31-33] is expected to have just one spin-allowed groundstate absorption transition for both octahedral and tetrahedral coordination in weak crystal fields (Dq/B $<2.6$ (octahedral) $\mathrm{Dq} / \mathrm{B}<2.0$ (tetrahedral)). Above these threshold 
values there are four spin-allowed transitions and the lowest energy transition is spinforbidden. In octahedral $\mathrm{V}^{+}$doped $\mathrm{ZnTe}$ one spin allowed transition absorption band is observed, centred at $2740 \mathrm{~nm}$, and is attributed to the ${ }^{5} \mathrm{~T}_{2}\left({ }^{5} \mathrm{D}\right) \rightarrow{ }^{5} \mathrm{E}\left({ }^{5} \mathrm{D}\right) \mathrm{nm}$ [39]. In octahedral $\mathrm{V}^{+}$doped $\mathrm{ZnSe}$ this transition occurred at $2604 \mathrm{~nm}[40]$. The $\mathrm{V}^{+}$configuration can therefore be discounted as we would expect to see only one absorption band if it resides in a weak crystal field or the characteristic narrow and long lifetime emission of a spin-forbidden transition in a high crystal field, neither of which are observed.

Based on these discussions the possible oxidation state of the vanadium ion is either $2+$ or $3+$ (or both). These are analyzed using the Tanabe-Sugano model in the next section.

\section{Tanabe-Sugano analysis of V:GLS}

\subsection{Introduction}

The Tanabe-Sugano model takes into account the interactions between two or more $3 \mathrm{~d}$ electrons in the presence of a crystal field. The free ion states are shown on the far left of a Tanabe-Sugano diagrams where $(\mathrm{Dq} / \mathrm{B}=0)$. The free ion states are governed by electron-electron interactions and so are labelled by ${ }^{2 \mathrm{~S}+1} \mathrm{~L}$ states (also called L-S terms) where $\mathrm{S}$ is the total spin angular momentum and $\mathrm{L}$ is the total orbital angular momentum [26]. The energy separation between the various ${ }^{2 \mathrm{~S}+1} \mathrm{~L}$ states is given in terms of the Racah parameters (A, B and C). These parameters describe the strength of the electrostatic interactions between multiple $3 \mathrm{~d}$ electrons [33]. Tanabe and Sugano calculated the energy matrices for each state of $3 \mathrm{~d}^{2}$ to $3 \mathrm{~d}^{5}$ ions in an ideal octahedral crystal field [31], their derivation was subsequently verified elsewhere [41]. These energy matrices can then be used to calculate how the ${ }^{2 \mathrm{~S}+1} \mathrm{~L}$ free ion levels split up, and vary, as a function of the ratio between the crystal field strength and the inter-electronic interaction (measured in Dq/B). Presented graphically, these functions are called Tanabe-Sugano diagrams and they have been used since their introduction in 1954 to interpret the spectra of transition metal ions in a variety of crystalline and glass hosts. Tanabe-Sugano diagrams take advantage of the fact that $\mathrm{C} / \mathrm{B}$ is almost independent of atomic number and the number of electrons, for all first row transition metal elements, $C / B \approx 4-5$ [31].

Cubic coordination can be thought of as representing two tetrahedral components. Hence the cubic crystal field interaction energy term has the same functional form as in a tetrahedral field but it is twice as large [33]. As a result of their small ionic radii in proportion to rare earth ions, transition metals are usually found in tetrahedral or octahedral coordination where as rare earths are often found in dodecahedral coordination [42]. Because of the relatively small ionic radii of the $\mathrm{V}^{2+}$ and $\mathrm{V}^{3+}$ ion and the relatively large ionic radii of the $\mathrm{S}^{2-}$ cation, cubic coordination in V:GLS is thought to be extremely unlikely. Low symmetry fields, such as tetragonal, cause a splitting of the energy terms. For example in tetrahedral $\mathrm{Cr}^{4+}: \mathrm{Y}_{2} \mathrm{SiO}_{5}$ with $\mathrm{C}_{3 v}$ symmetry the ${ }^{3} \mathrm{~T}_{1}\left({ }^{3} \mathrm{~F}\right)$ level splits into two components which were attributed to two closely spaced absorption peaks at 733 and $602 \mathrm{~nm}$ [43]. This sort of splitting is not evident in the absorption spectra of V:GLS so the data is analysed in terms of ideal octahedral or tetrahedral coordination.

The d-orbital splits into $t_{2}$ and e-orbitals in the presence of a crystal field. The various states are represented $\mathrm{as}_{2}{ }^{\mathrm{n}} \mathrm{e}^{\mathrm{m}}(\mathrm{n}+\mathrm{m}=\mathrm{N})$ where $\mathrm{N}$ is the number of electrons in 
the d-orbital. Tanabe and Sugano showed that it was unnecessary to calculate the energy matrices for $\mathrm{N}>5$ (which becomes very laborious) because of the simple relationship between configurations in the state $t_{2}{ }^{n} e^{m}$ and $t_{2}{ }^{6-n} e^{4-m}$. This simple relationship results from the equivalence of electrons and holes. It has been shown [32], that to obtain the full Hamiltonian energy matrices, $(-4 n+6 m) D q$ is added to the diagonal element in the state $\mathrm{t}_{2}{ }^{\mathrm{n}} \mathrm{e}^{\mathrm{m}}$. For the state $\mathrm{t}_{2}{ }^{6-\mathrm{n}} \mathrm{e}^{4-\mathrm{m}}$ this is $[-4(6-\mathrm{n})+6(4-\mathrm{m})] \mathrm{Dq}=-(-4 \mathrm{n}+6 \mathrm{~m}) \mathrm{Dq}$. It is also unnecessary to calculate the energy matrices for a tetrahedral field because the energy matrices for a $\mathrm{d}^{\mathrm{n}}$ ion in a tetrahedral field are the same as a $\mathrm{d}^{10-\mathrm{n}}$ ion in an octahedral field [33].

Each of the possible electronic configurations $\left(\mathrm{d}^{2}\right.$ or $\left.\mathrm{d}^{3}\right)$ and coordination (tetrahedral or octahedral) is now analysed with the Tanabe-Sugano model. The crystal field strength and other parameters are calculated using the energy $\left(\mathrm{cm}^{-1}\right)$ the two lowest spin-allowed transitions identified in Fig. 1 at $737 \mathrm{~nm}\left(13569 \mathrm{~cm}^{-1}\right)$ and $1100 \mathrm{~nm}(9091$ $\left.\mathrm{cm}^{-1}\right)$ and the spin-forbidden transition at $1000 \mathrm{~nm}\left(10000 \mathrm{~cm}^{-1}\right)$. The highest energy spin forbidden transition identified in Fig. 2 at $578 \mathrm{~nm}\left(17301 \mathrm{~cm}^{-1}\right)$ is not required for the calculation.

\subsection{Tetrahedral $d^{2}$ configuration}

The energy matrix for the ${ }^{3} \mathrm{~T}_{1}\left({ }^{3} \mathrm{~F},{ }^{3} \mathrm{P}\right)$ state of the tetrahedral $\mathrm{d}^{2}$ configuration can be found in ref [31], and is reproduced in table 1. The eigenvalues of the matrix in Table 1 give the energy terms of the ${ }^{3} \mathrm{~T}_{1}\left({ }^{3} \mathrm{~F}\right)$ and ${ }^{3} \mathrm{~T}_{1}\left({ }^{3} \mathrm{P}\right)$ states as a function of $\mathrm{Dq}$ and $\mathrm{B}$ obtained from the resulting diagonalised matrix elements. These are given in Eqs. (4) and (5), where E is the energy of the state in parentheses.

$$
\begin{aligned}
& E\left({ }^{3} \mathrm{~T}_{1}\left({ }^{3} \mathrm{~F}\right)\right)=\frac{1}{2}\left(-\mathrm{B}+6 \mathrm{Dq}-\sqrt{225 \mathrm{~B}^{2}-180 D q B+100 D q^{2}}\right) \\
& E\left({ }^{3} \mathrm{~T}_{1}\left({ }^{3} \mathrm{P}\right)\right)=\frac{1}{2}\left(-\mathrm{B}+6 \mathrm{Dq}+\sqrt{225 \mathrm{~B}^{2}-180 D q B+100 D q^{2}}\right)
\end{aligned}
$$

Dividing Eq. (4) by B and arranging in terms of $\mathrm{Dq} / \mathrm{B}$, as is necessary for Tanabe-Sugano diagrams, gives:

$$
E\left({ }^{3} \mathrm{~T}_{1}\left({ }^{3} \mathrm{~F}\right)\right) / \mathrm{B}=-\frac{1}{2}+3 \mathrm{Dq} / \mathrm{B}-\frac{1}{2} \sqrt{225-180 D q / B+100(D q / B)^{2}}
$$

Note that Eq. (6) is independent of $C$, in order to calculate $C$ the energy term for a spinforbidden energy level is needed. Table 2 gives the energy matrix for the ${ }^{1} \mathrm{E}\left({ }^{1} \mathrm{D},{ }^{1} \mathrm{G}\right)$ state. Diagonalizing the matrix in Table 2 and dividing by B gives:

$$
E\left({ }^{1} \mathrm{E}\left({ }^{1} \mathrm{G}\right)\right) / \mathrm{B}=\frac{1}{2}+2 C / B-2 \mathrm{Dq} / \mathrm{B}+\frac{1}{2} \sqrt{49+40 D q / B+400(D q / B)^{2}}
$$

and 


$$
E\left({ }^{1} \mathrm{E}\left({ }^{1} \mathrm{D}\right)\right) / \mathrm{B}=\frac{1}{2}+2 C / B-2 \mathrm{Dq} / \mathrm{B}-\frac{1}{2} \sqrt{49+40 D q / B+400(D q / B)^{2}}
$$

In Tanabe-Sugano diagrams, the energy term of the lowest energy level, in this case $\mathrm{E}\left({ }^{3} \mathrm{~A}_{2}\left({ }^{3} \mathrm{~F}\right)\right) / \mathrm{B}=-12 \mathrm{Dq} / \mathrm{B}-8$, is subtracted from the energy terms of all the energy levels, as has been done in Eqs. (9) - (12) for the energy levels of interest i.e., the three spinallowed energy levels and the lowest spin-forbidden energy level.

$$
\begin{gathered}
E\left({ }^{3} \mathrm{~T}_{2}\left({ }^{3} \mathrm{~F}\right)\right) / \mathrm{B}=10 D q / B \\
E\left({ }^{3} \mathrm{~T}_{1}\left({ }^{3} \mathrm{~F}\right)\right) / \mathrm{B}=7.5+15 \mathrm{Dq} / \mathrm{B}-\frac{1}{2} \sqrt{225-180 D q / B+100(D q / B)^{2}} \\
E\left({ }^{1} \mathrm{E}\left({ }^{1} \mathrm{D}\right)\right) / \mathrm{B}=8.5+2 C / B+10 \mathrm{Dq} / \mathrm{B}-\frac{1}{2} \sqrt{49+40 D q / B+400(D q / B)^{2}} \\
E\left({ }^{3} \mathrm{~T}_{1}\left({ }^{3} \mathrm{P}\right)\right) / \mathrm{B}=7.5+15 \mathrm{Dq} / \mathrm{B}+\frac{1}{2} \sqrt{225-180 D q / B+100(D q / B)^{2}}
\end{gathered}
$$

These energy terms are plotted in the Tanabe-Sugano diagram in Fig. 4 (a). They can now be used to calculate the crystal field strength $(\mathrm{Dq} / \mathrm{B})$ and $\mathrm{C} / \mathrm{B}$. Dq is known $(1 / 10$ the energy of the lowest spin allowed absorption transition) so $\mathrm{B}$ is calculated from the experimentally determined energies of the ${ }^{3} \mathrm{~T}_{2}\left({ }^{3} \mathrm{~F}\right)$ and ${ }^{3} \mathrm{~T}_{1}\left({ }^{3} \mathrm{~F}\right)$ energy levels and then solving their energy terms simultaneously for $\mathrm{B}$. The $\mathrm{C} / \mathrm{B}$ ratio is calculated by rearranging Eq. (8) to make C/B the subject as in Eq. (13).

$$
C / B=E\left({ }^{1} \mathrm{E}\left({ }^{1} \mathrm{D}\right)\right) / 2 \mathrm{~B}-5 \mathrm{Dq} / \mathrm{B}-4.25+\frac{1}{4} \sqrt{49+40 D q / B+400(D q / B)^{2}}
$$

The calculated values of $\mathrm{Dq} / \mathrm{B}, \mathrm{B}$, and $\mathrm{C} / \mathrm{B}$ are $1.85,485.12$ and 6.57 , respectively. The calculated value of $\mathrm{Dq} / \mathrm{B}$ is shown on Fig. 4 (a). It indicates a weak field site, which is consistent with the emission absorption and lifetime measurements. The calculated $\mathrm{C} / \mathrm{B}$ values are however slightly outside the allowed range of 4 to 5 . Nevertheless, tetrahedral $\mathrm{d}^{2}$ is a possible configuration of $\mathrm{V}$ :GLS.

\subsection{Octahedral $d^{2}$ configuration}

Using the procedure illustrated above we calculated the energy terms of interest for the octahedral $\mathrm{d}^{2}$ configuration from the appropriate energy matrices given in ref [31]. These energy terms are given in Eqs. (13) - (18) and plotted in the Tanabe-Sugano diagram in Fig. 4 (b). The values of $\mathrm{Dq} / \mathrm{B}, \mathrm{B}$ and $\mathrm{C} / \mathrm{B}$ were calculated using the same method as described for a $d^{2}$ tetrahedral ion. The energy term for the ${ }^{1} E\left({ }^{1} D\right)$ level was used to calculate $\mathrm{C} / \mathrm{B}$, as shown in Fig. 4 (b) this energy level is virtually indistinguishable from the ${ }^{1} \mathrm{~T}_{2}\left({ }^{1} \mathrm{D}\right)$ level. 


$$
\begin{gathered}
E\left({ }^{3} \mathrm{~T}_{2}\left({ }^{3} \mathrm{~F}\right)\right) / \mathrm{B}=-7.5+5 \mathrm{Dq} / \mathrm{B}+\frac{1}{2} \sqrt{225+180 D q / B+100(D q / B)^{2}} \\
E\left({ }^{3} \mathrm{~A}_{2}\left({ }^{3} \mathrm{~F}\right)\right) / \mathrm{B}=-7.5+15 \mathrm{Dq} / \mathrm{B}+\frac{1}{2} \sqrt{225+180 D q / B+100(D q / B)^{2}} \\
E\left({ }^{1} \mathrm{E}\left({ }^{1} \mathrm{D}\right)\right) / \mathrm{B}=1+2 C / B+5 \mathrm{Dq} / \mathrm{B}-\frac{1}{2} \sqrt{49-40 D q / B+400(D q / B)^{2}} \\
+\frac{1}{2} \sqrt{225+180 D q / B+100(D q / B)^{2}} \\
E\left({ }^{1} \mathrm{~T}_{2}\left({ }^{1} \mathrm{D}\right)\right) / \mathrm{B}=1+2 C / B-\frac{1}{2} \sqrt{49-20 D q / B+100(D q / B)^{2}} \\
+\frac{1}{2} \sqrt{225+180 D q / B+100(D q / B)^{2}} \\
E\left({ }^{3} \mathrm{~T}_{1}\left({ }^{3} \mathrm{P}\right)\right) / \mathrm{B}=\sqrt{225+180 D q / B+100(D q / B)^{2}}
\end{gathered}
$$

The calculated values of $\mathrm{Dq} / \mathrm{B}, \mathrm{B}$, and $\mathrm{C} / \mathrm{B}$ are $2.84,316.1$ and 12.61 , respectively. The calculated value of $\mathrm{Dq} / \mathrm{B}$ is much larger than that for $\mathrm{a} \mathrm{d}^{2}$ ion in tetrahedral coordination and the $\mathrm{C} / \mathrm{B}$ values are much larger that the allowed range of 4 to 5 and clearly are invalid. When the Tanabe-Sugano diagram is plotted with a valid C/B of 4.5, in Fig. 4 (b) it can be seen that the calculated Dq/B of 2.84 is in a strong field site, this is where the lowest energy transition is a spin forbidden transition. If this were the case we would expect to see narrow R-line emission, a long lifetime (in the ms to $\mathrm{s}$ regime) and have the characteristic weak and narrow spin forbidden absorption on the low energy side of the first spin-allowed absorption. In V:GLS the emission is very broad (FWHM $\sim 500 \mathrm{~nm}$ ) the lifetime is $\sim 30 \mu \mathrm{s}$ and spin-forbidden absorption is on the high energy side of the first spin allowed absorption peak. The octahedral $\mathrm{d}^{2}$ ion is therefore discounted as a possible configuration for V:GLS, with a high degree of confidence.

\subsection{Tetrahedral $d^{3}$ configuration}

The energy terms of the tetrahedral $\mathrm{d}^{3}$ configuration were calculated with the method described previously. These are plotted in the Tanabe-Sugano diagram in Fig. 4 (c). There was no solution found using the method previously described and when using the two lowest spin-allowed energy terms in both high and low field regions. The tetrahedral $\mathrm{d}^{3}$ ion is therefore discounted as a possible configuration for V:GLS. 


\subsection{Octahedral $d^{3}$ configuration}

The energy terms for the three lowest spin allowed energy levels of the octahedral $\mathrm{d}^{3}$ configuration the same for those calculated for the tetrahedral $\mathrm{d}^{2}$ configuration. However, the energy terms for the spin forbidden ${ }^{2} \mathrm{E}\left({ }^{2} \mathrm{G}\right)$ and ${ }^{2} \mathrm{~T}_{2}\left({ }^{2} \mathrm{G}\right)$ levels are different, but due to the complexity of the energy terms they were not considered in this work. These energy terms are plotted in Fig. 4 (d). As can been seen in Fig. 4 (d) the ${ }^{2} T_{1}\left({ }^{2} G\right)$ level lies close to the ${ }^{2} \mathrm{E}\left({ }^{2} \mathrm{G}\right)$ level so the spin forbidden transition could be caused by a transition to either or both of these energy levels. Because of this, $\mathrm{C} / \mathrm{B}$ was calculated using the energy term for both these energy levels. The calculated values of $\mathrm{Dq} / \mathrm{B}$ and $\mathrm{B}$ were 1.85 and 485.1, respectively. The $\mathrm{C} / \mathrm{B}$ values calculated using the energy terms for the ${ }^{2} \mathrm{E}\left({ }^{2} \mathrm{G}\right)$ and ${ }^{2} \mathrm{~T}_{2}\left({ }^{2} \mathrm{G}\right)$ levels were 4.55 and 4.25 , respectively. The $\mathrm{C} / \mathrm{B}$ parameter for the octahedral $\mathrm{d}^{3}$ configuration can also be calculated using an approximate formula given by Rasheed [44] and gives very similar results to our calculations. The value of $\mathrm{C} / \mathrm{B}$ calculated using for the octahedral $\mathrm{d}^{3}$ configuration are in the allowed range of 4 to 5 , unlike those calculated for the tetrahedral $\mathrm{d}^{2}$ configuration which was 6.57 . The calculated value of $\mathrm{Dq} / \mathrm{B}$ is shown in Fig. 4 (d) it indicates a weak field site which is consistent with the emission, absorption and lifetime measurements. In a tetrahedral $\mathrm{d}^{2}$ configuration the ${ }^{3} \mathrm{~A}_{2}\left({ }^{3} \mathrm{~F}\right) \rightarrow{ }^{3} \mathrm{~T}_{2}\left({ }^{3} \mathrm{~F}\right)$ transition is expected to be significantly weaker than the other two spin-allowed transitions because it is only magnetic dipole allowed [7, 45], however this is not evident from the derivative absorption and PLE spectra of V:GLS, this indicates that V:GLS, may not have a tetrahedral $\mathrm{d}^{2}$ configuration.

The above arguments indicate that the octahedral $\mathrm{d}^{3}$ configuration is more representative of $\mathrm{V}$ :GLS than the tetrahedral $\mathrm{d}^{2}$ configuration. We therefore propose that the three spin allowed absorption bands identified in Figs. 1 and 2 at 1100,737 and 578 $\mathrm{nm}$ are due to ${ }^{4} \mathrm{~A}_{2}\left({ }^{4} \mathrm{~F}\right) \rightarrow{ }^{4} \mathrm{~T}_{2}\left({ }^{4} \mathrm{~F}\right),{ }^{4} \mathrm{~A}_{2}\left({ }^{4} \mathrm{~F}\right) \rightarrow{ }^{4} \mathrm{~T}_{1}\left({ }^{4} \mathrm{~F}\right)$ and ${ }^{4} \mathrm{~A}_{2}\left({ }^{4} \mathrm{~F}\right) \rightarrow{ }^{4} \mathrm{~T}_{1}\left({ }^{4} \mathrm{P}\right)$ transitions respectively and the spin-forbidden transition at $1000 \mathrm{~nm}$ is attributed to the ${ }^{4} \mathrm{~A}_{2}\left({ }^{4} \mathrm{~F}\right) \rightarrow$ ${ }^{2} \mathrm{E}\left({ }^{2} \mathrm{G}\right)$ or ${ }^{4} \mathrm{~A}_{2}\left({ }^{4} \mathrm{~F}\right) \rightarrow{ }^{2} \mathrm{~T}_{2}\left({ }^{2} \mathrm{G}\right)$ transition. The emission peaking at $1500 \mathrm{~nm}$ is due to the ${ }^{4} \mathrm{~T}_{2}\left({ }^{4} \mathrm{~F}\right) \rightarrow{ }^{4} \mathrm{~A}_{2}\left({ }^{4} \mathrm{~F}\right)$ transition. These energy level assignments were used to create the energy level diagram for V:GLS shown in Fig. 5. The positions and widths of the energy levels was taken from the absorption and excitation data presented in Figs. 1 and 2. The width of the ${ }^{2} \mathrm{E}\left({ }^{2} \mathrm{G}\right)$ energy level is an estimate.

To put these findings in a structural context, other authors have proposed that GLS consists of a covalent network of $\mathrm{GaS}_{4}$ tetrahedra, inter-dispersed by essentially ionic La-S channels [46-48]. In addition some $\mathrm{GaS}_{4}$ tetrahedra with a negative charge are formed from the $\mathrm{Ga}$ being bonded to one sulphur anion $\left(\mathrm{S}^{2-}\right)$ to produce "sulphide negative cavities". Oxygen anions $\left(\mathrm{O}^{2-}\right)$ in the glass will similarly produce negatively charged $\mathrm{GaS}_{3} \mathrm{O}$ tetrahedra or "oxide negative cavities". These negative ionic cavities form some reception sites for $\mathrm{La}^{3+}$ ions, which act as charge compensators for these negative charges.[24, 47] Dopant ions in glasses are generally expected to enter substitutionally for network modifier cations [33]. The main network modifier in GLS is $\mathrm{La}^{3+}$ [47] which is 8-fold coordinated to sulphur with an undetermined symmetry [46]. The ionic radii of $\mathrm{La}^{3+}, \mathrm{V}^{2+}$ and $\mathrm{V}^{3+}$ are $1.03,0.79$ and $0.64 \AA$, respectively [49]. We tentatively propose that $\mathrm{V}$ substitute for $\mathrm{La}^{3+}$ and is sulphide coordinated. The matching, in terms of ionic radii and coordination number, between octahedral $\mathrm{V}^{2+}$ and its proposed $\mathrm{La}^{3+}$ reception site is not ideal. However, out of the possible vanadium configurations 
(octahedral or tetrahedral $\mathrm{V}^{2+}$ or $\mathrm{V}^{3+}$ ), octahedral $\mathrm{V}^{2+}$ is clearly the most likely to substitute into the proposed reception site.

\section{Conclusions}

Absorption measurements of V:GLS unambiguously identified one absorption band at $1100 \mathrm{~nm}$, and two further higher energy absorption bands that could not be fully resolved. Derivative analysis of the absorption spectrum identified a spin-forbidden absorption transition at $1000 \mathrm{~nm}$ and was able to resolve an additional narrow absorption band at $737 \mathrm{~nm}$. However, the highest energy absorption band could not be resolved by derivative analysis due to the close proximity of the host electronic absorption edge. PLE measurements were able to resolve three absorption bands. Deconvolution of the spectra into three Gaussians revealed peaks at 1154, 766 and $578 \mathrm{~nm}$. The PLE peaks undergo a small red-shift in comparison to the absorption peaks. The PLE peaks are shifted to longer wavelengths than the absorption peaks because excitation at longer wavelengths than the absorption peak shifts the PL to longer wavelengths, where there will be less reabsorption of the emission. XPS measurements indicated the presence of vanadium in a broad range of oxidation states from $\mathrm{V}^{+}$to $\mathrm{V}^{5+}$. Excitation into each of the three absorption bands identified by PLE measurements produced the same characteristic emission spectrum, peaking at $1500 \mathrm{~nm}$ with a FWHM of $\sim 500 \mathrm{~nm}$. The decay lifetime and decay profile were also similar for each excitation wavelength. This was a strong indication that only one of the vanadium oxidation states is responsible for the observed absorption bands. Out of the possible vanadium oxidation states, only $\mathrm{V}^{2+}$ and $\mathrm{V}^{3+}$ is expected to exhibit three spin-allowed transitions. Tanabe-Sugano analysis indicates that out of the possible configurations of coordination and oxidation state only tetrahedral $\mathrm{V}^{3+}$ and octahedral $\mathrm{V}^{2+}$ has a crystal field strength in the expected low field region. Of these configurations only octahedral $\mathrm{V}^{2+}$ has a $\mathrm{C} / \mathrm{B}$ value in the expected range of 4 to 5 . The configuration of the optically active vanadium ion in $\mathrm{V}: \mathrm{GLS}$ is therefore proposed to be octahedral $\mathrm{V}^{2+}$. The crystal field parameters $(\mathrm{Dq} / \mathrm{B}, \mathrm{B}$ and $\mathrm{C} / \mathrm{B})$, calculated for the proposed configuration were $1.85,485.1$ and 4.55 , respectively. Octahedral $\mathrm{V}^{2+}$ enters the glass network by substituting for $\mathrm{La}^{+}$. The detailed explanation of the Tanabe-Sugano model and the arguments used to determine the oxidation state in this work may be useful to other researchers tackling similar problems. 
[1] I. Abdulhalim, C. N. Pannell, R. S. Deol, D. W. Hewak, G. Wylangowski and D. N. Payne, J. Non-Cryst. Solids 164-166 (1993), p. 1251.

[2] T. Schweizer, D. W. Hewak, B. N. Samson and D. N. Payne, Opt. Lett. 21 (1996), p. 1594.

[3] T. Schweizer, B.N.Samson, J.R.Hector, W.S.BrocklesbyD.W.Hewak and D.N.Payne, Infrared Phys. Techn. 40 (1999), p. 329.

[4] M. A. Hughes, R. J. Curry and D. W. Hewak, J. Opt. Soc. Am. B: Opt. Phys. 25 (2008), p. 1458.

[5] M. A. Hughes, T. Akada, T. Suzuki, Y. Ohishi and D. W. Hewak, Opt. Express 17 (2009), p. 19345.

[6] C. Anino, J. Thery and D. Vivien, Opt. Mater. 8 (1997), p. 121.

[7] X. Feng, Opt. Mater. 20 (2002), p. 63.

[8] T. Suzuki, G. S. Murugan and Y. Ohishi, Appl. Phys. Lett. 86 (2005), p. 131903.

[9] M. Hughes, H. Rutt, D. Hewak and R. Curry, Appl. Phys. Lett. 90 (2007), p. 031108 .

[10] T. C. O'Haver and T. Begley, Anal. Chem. 53 (1981), p. 1876.

[11] W. L. Butler and D. W. Hopkins, Photochem. Photobiol. 14 (1970), p. 439.

[12] R. K. Brow, J. Am. Ceram. Soc. 70 (1987), p. 129.

[13] W. D. Johnstone, J. Am. Ceram. Soc. 48 (1965), p. 608.

[14] M. A. Noginov, J. Appl. Phys. 91 (2002), p. 569.

[15] A. M. Malyarevich, Appl. Phys. B-Lasers Opt. 67 (1998), p. 555.

[16] A. G. Khudoleev and N. M. Bokin, J. Appl. Spectrosc. 14 (1971), p. 75.

[17] Z. Goldschmidt, W. Low and M. Foguel, Phys. Lett. 19 (1965), p. 17.

[18] D. Briggs, Handbook of X-ray and ultraviolet photoelectron spectroscopy, (1977).

[19] D. Briggs, Electron spectroscopy: Theory, Techniques and Applications, (1978).

[20] P. C. Schultz, J. Am. Ceram. Soc. 57 (1974), p. 309.

[21] G. A. Sawatzky and D. Post, Phys. Rev. B 20 (1979), p. 1546.

[22] H. Farah and M. Brungs, J. Mater. Sci. 38 (2003), p. 1885.

[23] H. Farah, J. Am. Ceram. Soc. 91 (2008), p. 3915.

[24] T. Schweizer, F. Goutaland, E. Martins, D. W. Hewak and W. S. Brocklesby, J. Opt. Soc. Am. B: Opt. Phys. 18 (2001), p. 1436.

[25] A. Mekki, D. Holland, C. F. McConville and M. Salim, J. Non-Cryst. Solids 208 (1996), p. 267.

[26] J. Garcia-Sole, L. E. Bausa and D. Jaque, An introduction to the Optical Spectroscopy of Inorganic Solids, Wiley, Chichester (2005).

[27] A. Belletti, R. Borromei, E. Cavalli and L. Oleari, Eur. J. Solid State Inorg. Chem. 35 (1998), p. 483.

[28] T. C. Brunold, H. U. Gudel and A. A. Kaminskii, Chem. Phys. Lett. 271 (1997), p. 327.

[29] J. P. Meyn, T. Danger, K. Petermann and G. Huber, J. Lumin. 55 (1993), p. 55.

[30] M. Yamaga, B. Henderson and Y. Yosida, Phys. Rev. B 51 (1995), p. 3438.

[31] Y. Tanabe and S. Sugano, J. Phys. Soc. Jpn. 9 (1954), p. 753.

[32] S. Sugano, Y. Tanabe and H. Kamimura, Multiplets of transition-metal ions in crystals Academic Press, New York (1970).

[33] B. Henderson and G. F. Imbusch, Optical spectroscopy of inorganic solids, Oxford University Press, Oxford (1989). 
[34] V. P. Mikhailoz and N. V. Kuleshove, Opt. Mater. 2 (1993), p. 267.

[35] K. V. Yumashev, N. V. Kuleshov and A. M. Malyarevich, J. Appl. Phys. 80 (1996), p. 4782.

[36] R. Moncorge and T. Benyattou, Phys. Rev. B 37 (1988), p. 9177.

[37] W. Knierim, A. Honold, U. Brauch and U. Durr, J. Opt. Soc. Am. B: Opt. Phys. 3 (1985), p. 119.

[38] U. Brauch and U. Durr, Opt. Commun. 55 (1985), p. 35.

[39] P. Peka, M. U. Lehr and H.-J. Schulz, Phys. Rev. B 53 (1996), p. 1907.

[40] G. Goetzt, U. W. Pohlt and H.-J. Schulzi, J. Phys.: Condens. Matter 4 (1992), p. 8253.

[41] J. S. Griffith, Theory of transition metal ions, Cambridge University Press, London (1961).

[42] H. P. Christensen and H. P. Jenssen, IEEE J. Quantum Electron. 18 (1982), p. 1197.

[43] C. Deka, M. Bass, B. H. T. Chai and Y. Shimony, J. Opt. Soc. Am. B: Opt. Phys. 10 (1993), p. 1499.

[44] F. Rasheed, K. P. O'Donnell, B. Henderson and D. B. Hollis, J. Phys.: Condens. Matter 3 (1990), p. 1915.

[45] S. Kuck, K. Peterman, U. Pohlmann and G. Huber, J. Lumin. 68 (1996), p. 1.

[46] R. Asal, P. E. Rivers and H. N. Rutt, J. Phys.: Condens. Matter 9 (1997), p. 6217.

[47] S. Benazeth, M. H. Tuilier, H. Dexpert, M. Guittard and D. Carre, Journal de Physique C8 (1986), p. 419.

[48] S. Benazeth, M. H. Tuilier, A. M. Loireau-Lozac'h, H. Dexpert, P. Lagarde and J. Flahaut, J. Non-Cryst. Solids 110 (1989), p. 89.

[49] R. D. Shannon, Acta Crystallogr. Sect. A 32 (1976), p. 751.

Fig. 1. Absorption spectra of undoped and $0.1 \%$ vanadium doped GLS. The second derivatives of these spectra are also shown

Fig. 2. PLE spectrum of $0.1 \%$ vanadium doped GLS fitted with three Gaussians. PL spectra at three different excitation wavelengths are also shown.

Fig. 3. X-ray photoelectron spectrum of $1 \%$ vanadium doped GLS. The inset shows a close-up of the vanadium peaks.

Fig. 4. Tanabe-Sugano diagrams of the tetrahedral $d^{2}(a)$, octahedral $d^{2}(b)$, tetrahedral $d^{3}$ (c) and octahedral $\mathrm{d}^{3}$ (d) configurations. Dashed, vertical lines show the calculated values of $\mathrm{Dq} / \mathrm{B}$.

Fig. 5. Energy level diagram of vanadium 2+ doped GLS 
Table 1 energy matrix for the ${ }^{3} \mathrm{~T}_{1}\left({ }^{3} \mathrm{~F},{ }^{3} \mathrm{P}\right)$ state.

\begin{tabular}{cc}
\hline $\mathbf{t}_{2}{ }^{2}$ & $\mathbf{t}_{\mathbf{2}} \mathbf{e}$ \\
\hline$-5 \mathrm{~B}+8 \mathrm{Dq}$ & $6 \mathrm{~B}$ \\
$6 \mathrm{~B}$ & $4 \mathrm{~B}-2 \mathrm{Dq}$ \\
\hline
\end{tabular}

Table 2 energy matrix for the ${ }^{1} \mathrm{E}\left({ }^{1} \mathrm{D},{ }^{1} \mathrm{G}\right)$ state.

\begin{tabular}{cc}
\hline $\mathbf{t}_{\mathbf{2}}{ }^{2}$ & $\mathbf{e}^{2}$ \\
\hline $\mathrm{B}+2 \mathrm{C}+8 \mathrm{Dq}$ & $-2 \sqrt{3 \mathrm{~B}}$ \\
$-2 \sqrt{ } 3 \mathrm{~B}$ & $2 \mathrm{C}-12 \mathrm{Dq}$ \\
\hline
\end{tabular}




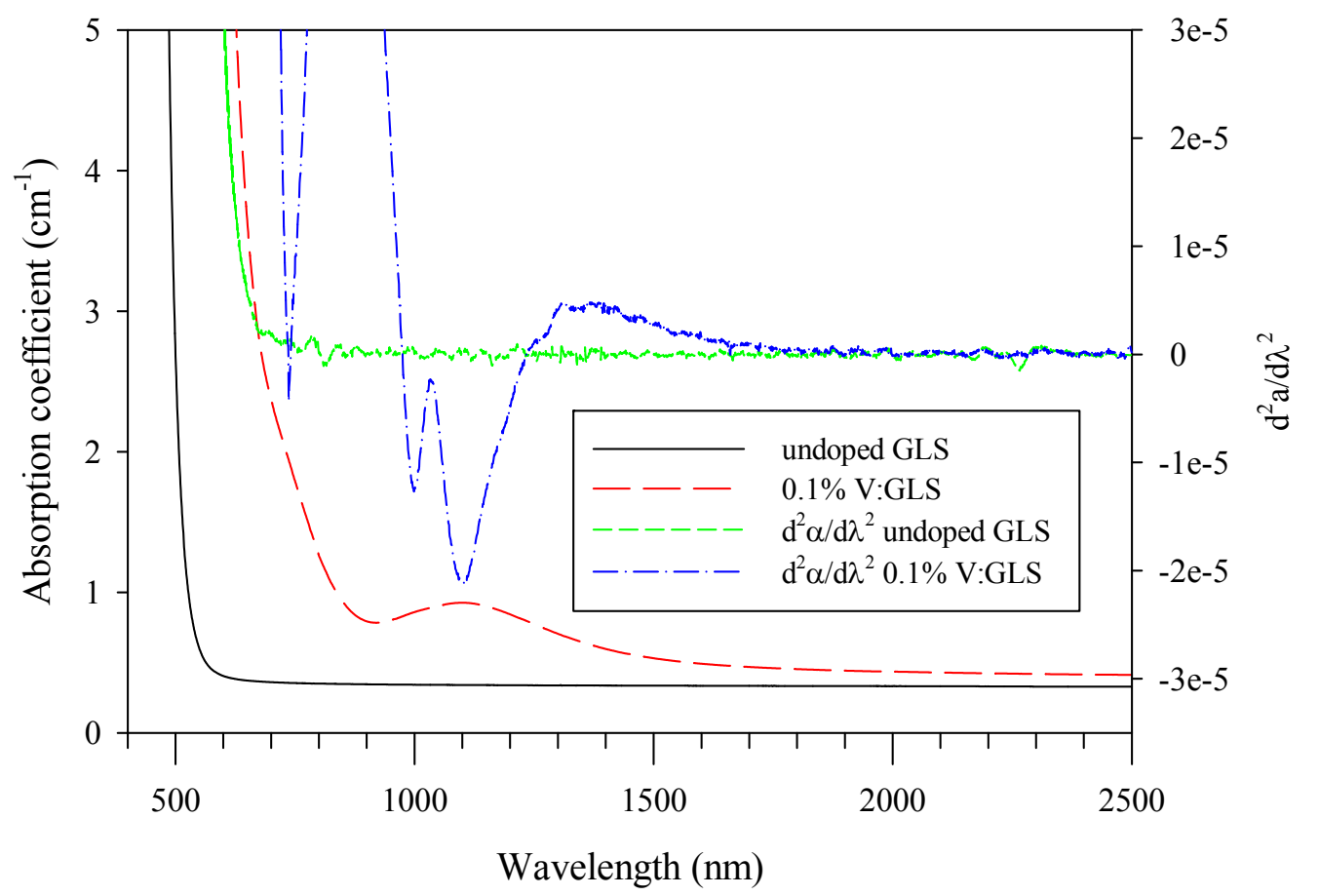

Fig. 1 Absorption spectra of undoped and $0.1 \%$ vanadium doped GLS. The second derivatives of these spectra are also shown 


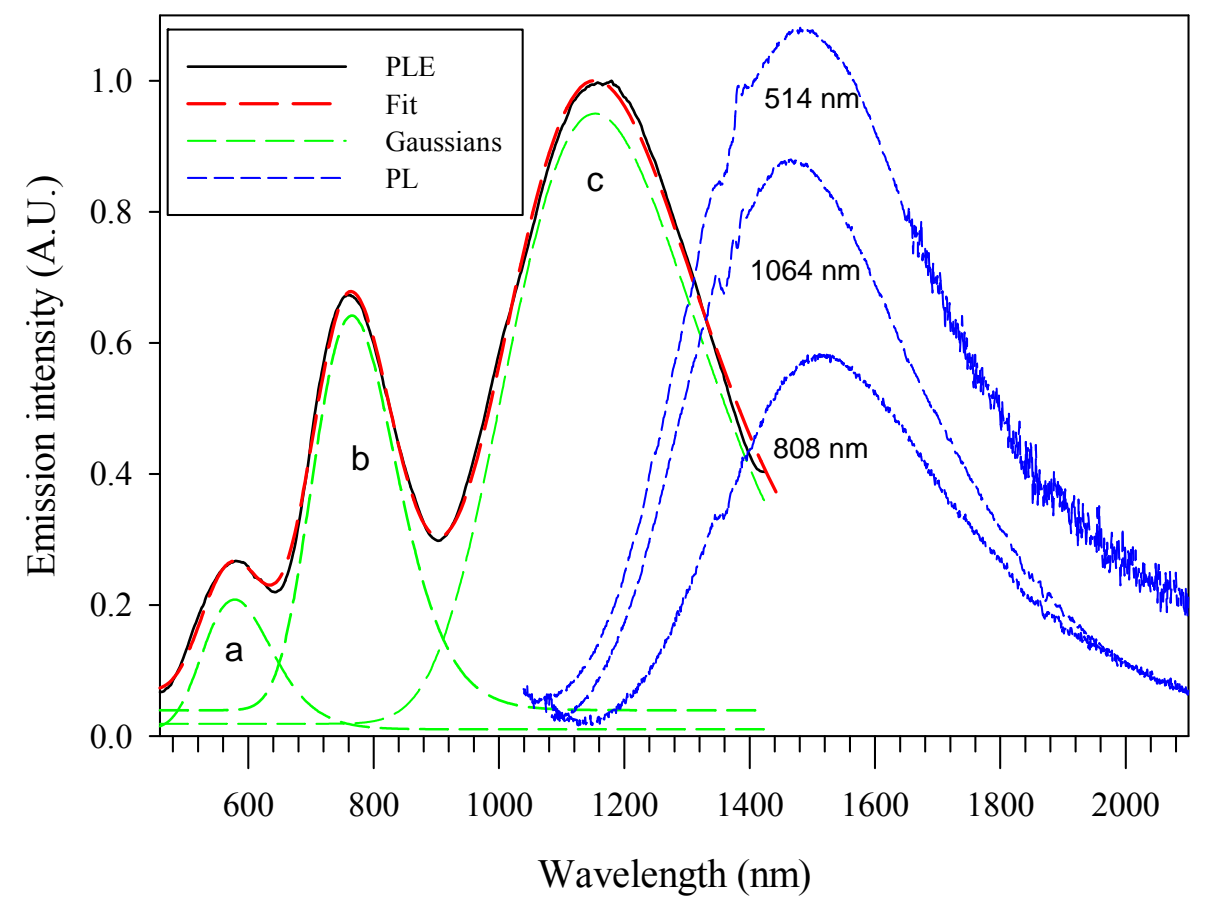

Fig. 2 PLE spectrum of $0.1 \%$ vanadium doped GLS fitted with three Gaussians. PL spectra at three different excitation wavelengths are also shown. 


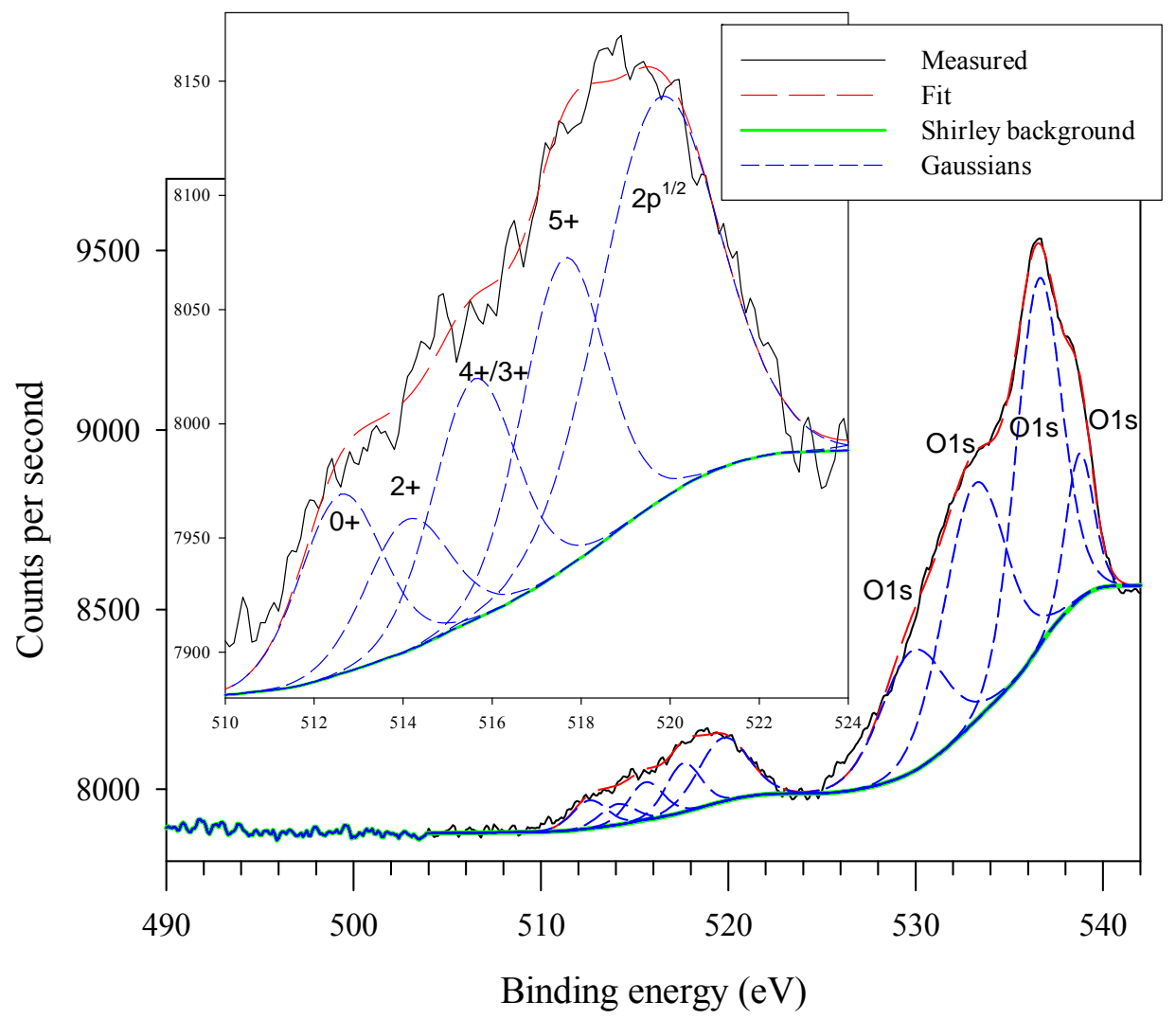

Fig. 3. X-ray photoelectron spectrum of $1 \%$ vanadium doped GLS. The inset shows a close-up of the vanadium peaks. 

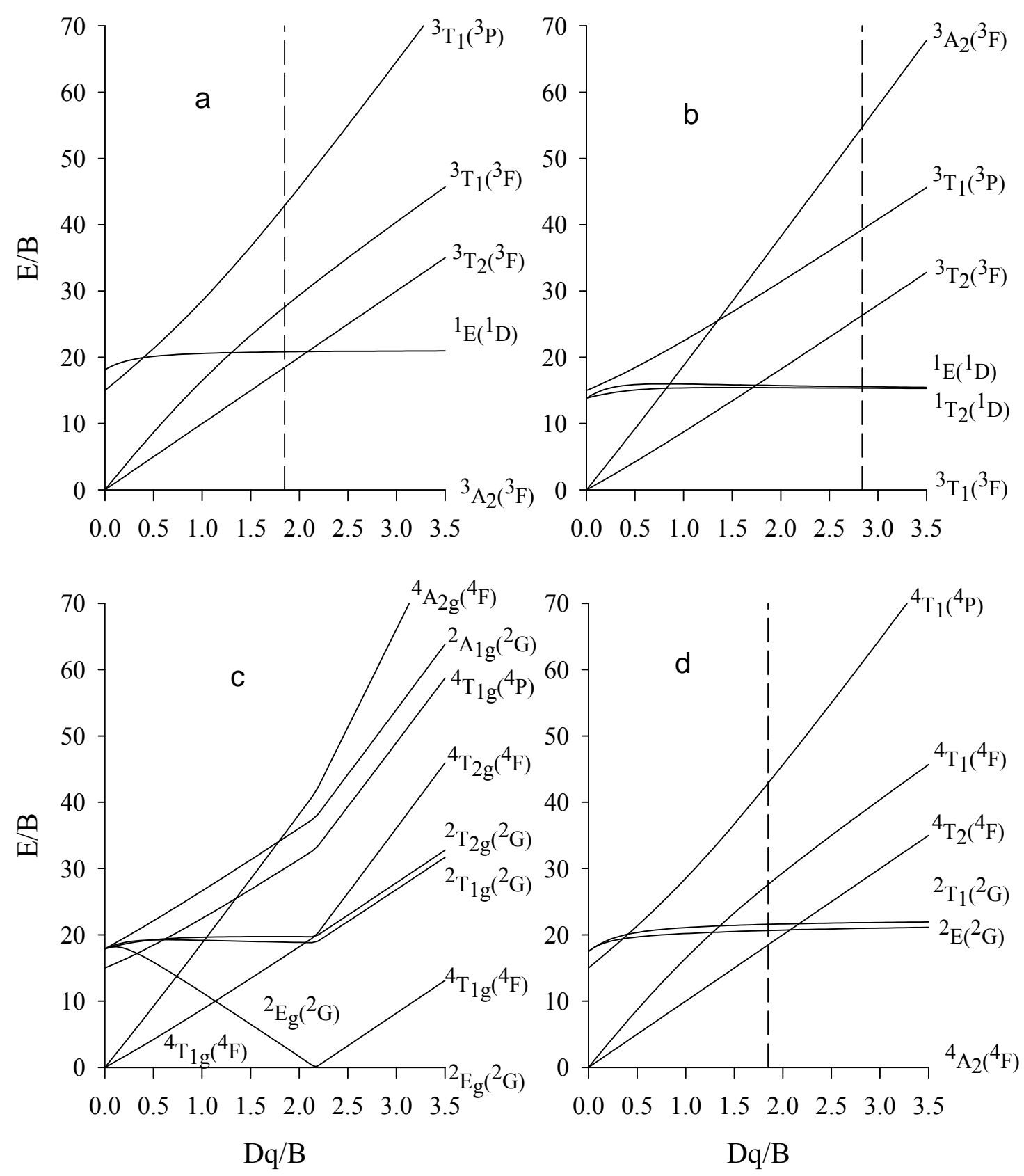

Fig. 4 Tanabe-Sugano diagrams of the tetrahedral $d^{2}(a)$, octahedral $d^{2}(b)$, tetrahedral $d^{3}$ (c) and octahedral $\mathrm{d}^{3}$ (d) configurations. Dashed, vertical lines show the calculated values of $\mathrm{Dq} / \mathrm{B}$. 


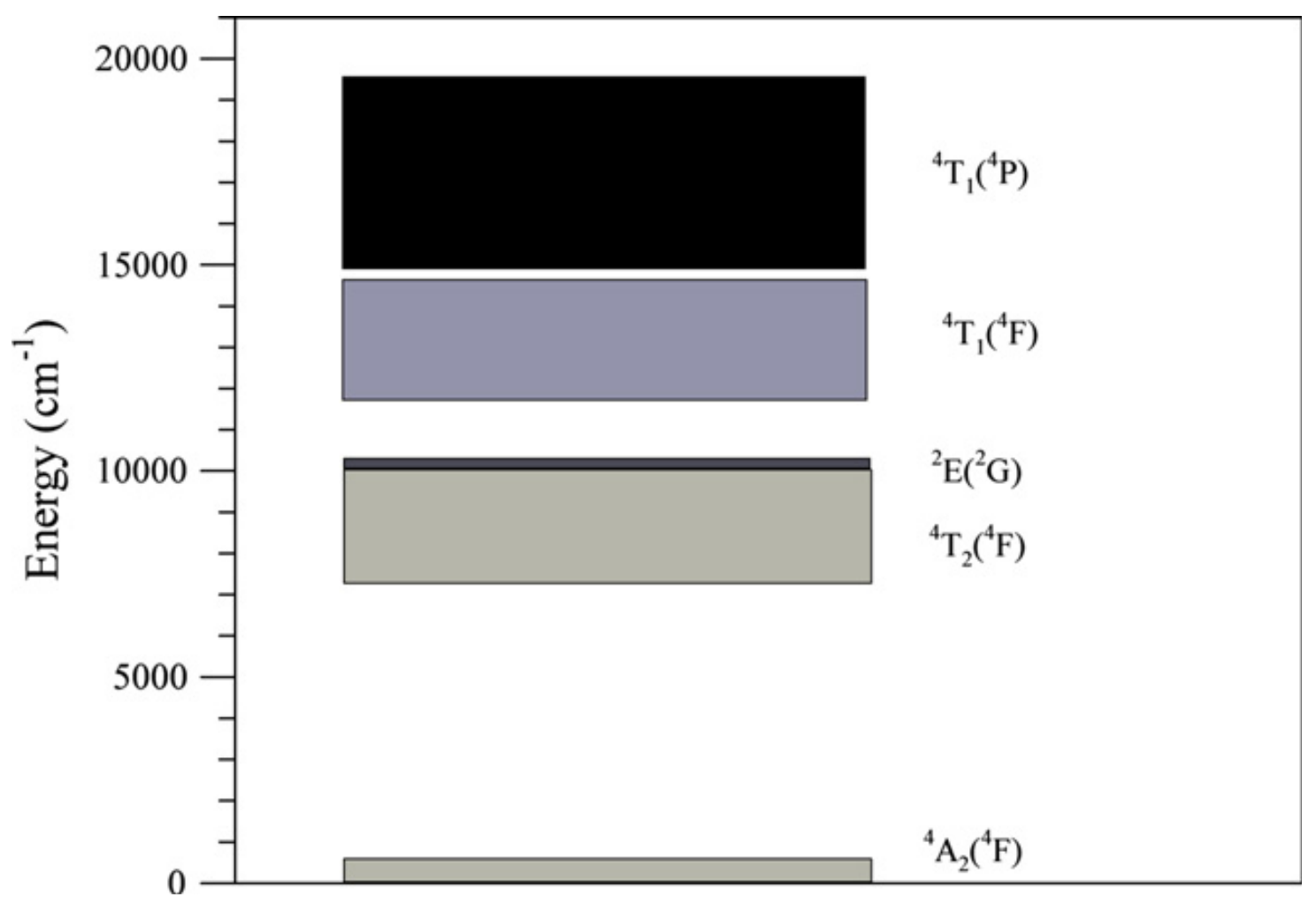

Fig. 5. Energy level diagram of vanadium $2+$ doped. 